\title{
Price for environmental neutrino-superluminality
}

\author{
Gia Dvali ${ }^{a, b, c, d}$ and Alexander Vikman ${ }^{a}$ \\ ${ }^{a}$ Theory Division, CERN, \\ 1211 Geneva 23, Switzerland \\ ${ }^{b}$ Arnold Sommerfeld Center, Department für Physik, Ludwig-Maximilians Universität München, \\ Theresienstr. 3\%, 80333 München, Germany \\ ${ }^{c}$ Max-Plank-Institut für Physik, \\ Föhringer Ring 6, 80805 München, Germany \\ ${ }^{d}$ Center for Cosmology and Particle Physics, Department of Physics, New York University, \\ 4 Washington Place, New York, NY 10003, U.S.A. \\ E-mail: georgi.dvali@cern.ch, alexander.vikman@cern.ch
}

ABSTRACT: We ask whether the recent OPERA results on neutrino superluminality could be an environmental effect characteristic of the local neighborhood of our planet, without the need of violation of the Poincaré-invariance at a fundamental level. We show, that model-indepenently, such a possibility implies the existence of new gravitational degrees of freedom. Namely, this explanation requires the existence of a new spin-2 field of a planetary Compton wave-length that is coupled to neutrinos and the rest of the matter asymmetrically, both in the magnitude and in the sign. Sourced by the earth this field creates an effective metric on which neutrinos propagate superluminally, whereas other species are much less sensitive to the background. Such a setup, at an effective field theory level, passes all immediate phenomenological tests, but at the expense of sacrificing calculability for some of the phenomena that are under perturbative control in ordinary gravity. The natural prediction is an inevitable appearance of a testable long-range gravitytype fifth force. Despite phenomenological viability, the sign asymmetry of the coupling we identify as the main potential obstacle for a consistent UV-completion. We also discuss the possible identification of this field with a Kaluza-Klein state of an extra dimension in which neutrino can propagate.

Keywords: Beyond Standard Model, Neutrino Physics, Space-Time Symmetries

ARXIV EPRINT: 1109.5685 
This note is inspired by recent results by OPERA [1] about possible evidence for superluminal propagation of neutrinos. Needless to say, discovery of superluminality would require major rethinking of our understanding of principles of relativity. In this note we shall assume that OPERA results indeed point to superluminality of neutrinos and ask what minimal changes in the Standard Model physics could accommodate such a phenomenon.

An immediate challenge is to reconcile OPERA results with the absence of analogous observations for superluminal propagation for supernova neutrinos.

One possible approach would be to suggest violation of the Poincaré invariance at the fundamental level through some energy-dependent operators that would result in superluminal propagation in an energy-dependent way, see e.g. [2, 3]. This is a logical possibility, but we shall take a different root.

We shall not postulate any violation of the Poincaré symmetry at the fundamental level. Instead, we shall ask whether the effect is environmental and takes place in the local neighborhood of the earth. We shall show that such an explanation, under the assumption of a calculable weakly-coupled physics, leads us to inevitability of existence of a new gravitytype force, asymmetrically-coupled to neutrinos and to the heavy matter, such as nucleons.

We shall structure our discussion in the following way. We shall first show that the environmental superluminality can be explained by introduction of the above-mentioned new gravity-type force. Then we will prove that this is the only possible environmental explanation under the assumption of the weak coupling and calculability of the neutrino propagation. This new force in its calculability range is compatible with all the immediate phenomenological constraints. But, the phenomenological price to pay is a very low strong coupling scale, which sacrifices calculability for some systems for which the ordinary gravity would be weakly coupled and is under control. It is extremely important that appearance of such a low cutoff is an inevitable consequence of any environmental explanation of OPERA results.

Thus, let us accomplish the first goal by postulating existence of a new light bosonic degree of freedom with the Compton wavelength of the order of planetary distances. The role of this degree of freedom is simple. We assume that this field is sourced by earth and creates a classical background to which neutrino is coupled. Neutrino then propagates through an effective metric that speeds it up.

For concreteness, we shall illustrate this idea on an example of a new massive spin-2 degree of freedom, $h_{\mu \nu}$, coupled to neutrino in the following way,

$$
\left(\eta_{\mu \nu}+\frac{h_{\mu \nu}}{M_{*}}\right) \bar{\nu} \gamma^{\mu} \partial^{\nu} \nu
$$

For simplicity of presentation, we shall treat neutrino as massless. We view the above expression as an effective low energy coupling in which all the heavy weak-scale physics has been integrated out. As a result, neutrino sees the following effective metric ${ }^{1}$

$$
g_{\mu \nu}^{(\nu)}=\eta_{\mu \nu}+\frac{h_{\mu \nu}}{M_{*}} .
$$

\footnotetext{
${ }^{1}$ For neutrino this metric plays a role of the contravariant metric in standard GR, while signal propagates in the covariant metric which is inverse to the one in (2). Throughout the paper we raise and lower indices with $\eta^{\mu \nu}$ and $\eta_{\mu \nu}$ respectively.
} 
The scale $M_{*}$ sets the strength of the coupling. Thus, the interaction of $h_{\mu \nu}$ with neutrino is similar to linear gravity. However, for us $h_{\mu \nu}$ is just another massive spin-2 field, not necessarily of any geometric origin. Correspondingly, the scale $M_{*}$ is not the Planck mass, and its value will be constrained below from the OPERA data. On a non-trivial background with $h_{\mu \nu}$ field the above coupling effectively amounts to changing the anti-commutation relation of effective gamma matrices to a new metric.

We now have to specify the coupling of $h_{\mu \nu}$ to other Standard Model particles. Of main importance is the coupling with the species that give dominant contribution into the earth's mass, such as, nucleons. Since we are not making any assumptions about the gravitational origin of $h_{\mu \nu}$, its couplings to the latter states do not have to obey the equivalence principle. The simplest possibility, however, is when the coupling to the rest of the species is universal and is through energy-momentum tensors,

$$
\frac{h_{\mu \nu}}{M} T^{\mu \nu},
$$

where $T_{\mu \nu}$ should not include neutrino. Since in our analysis we shall work at the level of very low energy effective theory, $T_{\mu \nu}$ can be directly taken to be an effective energy momentum tensor of the earth.

In order to complete our analysis, we will need a Lagrangian for $h_{\mu \nu}$. For the range of energies and distances of our interests the linearized analysis will be fully sufficient and reliable. Therefore, we shall restrict ourselves with the linear action, which is uniquely fixed to be of the Pauli-Fierz form,

$$
h^{\mu \nu} \mathcal{E} h_{\mu \nu}+m^{2}\left(h_{\mu \nu} h^{\mu \nu}-h_{\mu}^{\mu} h_{\nu}^{\nu}\right)
$$

where $\mathcal{E} h_{\mu \nu}$ is the linearized Einstein's tensor.

Non-linear interactions shall play no role in our analysis. We are fully aware of subtleties of non-linearities, since they usually result into low cutoffs. It is not our goal to extend the theory beyond these cutoffs, and we shall safely stay below it. Even assuming a most conservative case, the scale of non-linearities (a so-called Vainshtein scale [4]) for our choice of parameters dictated by OPERA, appears way beyond the range of our interest. As a result, we can perform a fully reliable computation in a linear regime.

Thus, the effective Lagrangian we work with represents the sum of the three terms given in equations (1), (3) and (4). As we shall see, in order to explain OPERA results, the scales $M$ and $M_{*}$ must be above and below the Planck mass respectively. This choice results in the following situation. Mass of the earth sources $h_{\mu \nu}$ and creates a local classical field. This field will have a negligible effect on a local gravitational background seen by all the particles except neutrino. The latter shall feel the $h_{\mu \nu}$ background much stronger and as a result become slightly superluminal.

In order to see this, let us find a static background of $h_{\mu \nu}$ created by the earth. This is provided by the solution of the linearized equation

$$
\left(-\Delta+m^{2}\right) h_{\mu \nu}=\frac{1}{M}\left(T_{\mu \nu}-\frac{1}{3}\left(\eta_{\mu \nu}+\frac{\partial_{\mu} \partial_{\nu}}{m^{2}}\right) T\right)
$$


in which $T_{\mu \nu}$ is taken as a non-relativistic spherical source of the earth's mass, $M_{E}$. The result for time and space components at distance $r \ll m^{-1}$ is,

$$
h_{00}=\frac{2}{12 \pi} \eta_{00} \frac{M_{E}}{M r}, \quad h_{i j}=-\frac{1}{12 \pi} \eta_{i j} \frac{M_{E}}{M r},
$$

where the contribution proportional to total derivatives has been neglected due to conservation of the probe neutrino source. Correspondingly the effective metric in which neutrino propagates is

$$
g_{00}^{(\nu)}=\left(\left(1-\frac{1}{3} \epsilon\right)+\epsilon\right) \eta_{00}, \quad g_{i j}^{(\nu)}=\left(1-\frac{1}{3} \epsilon\right) \eta_{i j},
$$

where we have introduced a notation ${ }^{2}$

$$
\epsilon \equiv \frac{M_{E}}{4 \pi M_{*} M r} .
$$

Clearly the property of superluminality is determined by the sign of $\epsilon$, which depends on the relative sign of $M$ and $M_{*}$. When the sign is negative, $\epsilon<0$, the propagation is superluminal.

The OPERA results correspond to $\epsilon \sim 10^{-5}$. Then taking the distance of the order of the earth-radius $r \sim 10^{8} \mathrm{~cm}$, we get that the OPERA observation can be reproduced by

$$
M_{*} M \sim 10^{-4} M_{P}^{2} .
$$

An independent important constraint on the scale $M$ is coming from the absence of any observable long-range fifth force of gravity-type. Depending on the precise nature of couplings this fact implies the constraint on $M$ in a wide window, $M^{2} / M_{P}^{2}>10^{4}-10^{12}$. This bound follows from applying the experimental fifth force bounds [5] to the case of additional graviton(s) with Compton wave-length of the earth's radius derived in details in [6], where such gravitons where motivated by the studies of earth-size extra dimension. These results can be directly applied to our case. The upper edge of the interval would take place in case of maximal violation of the equivalence principle.

Assuming universality, and combining the two bounds, we get, $M_{*} \sim 10^{-6} M_{P}$ and $M \sim 10^{2} M_{P}$.

Coming back to the consistency of our estimate, let us note that for such values of the parameters, even in the worst possible scenario (in which no weakly-coupled completion exists before the strong coupling scale) the upper bound on the Vainshtein's radius (distance at which non-linearities become important) is at $R_{V} \sim\left(\left(M_{E} / M^{2}\right) m^{-4}\right)^{1 / 5}$. For $M \sim$ $10^{2} M_{P}$ and $\mathrm{m}^{-1} \sim 10^{8} \mathrm{~cm}$ we get $R_{V} \sim 10^{5} \mathrm{~cm}$, which is way inside the earth's radius. Thus for our purposes, the linear regime is a very good approximation and can be trusted.

We see that in order to account for the OPERA result, the hierarchy of couplings can be relatively mild. Note, that such a hierarchy is radiatively stable, since $1 / M_{*}$ coupling of $h_{\mu \nu}$ to hadrons will be communicated only at the two-loop level, being suppressed by powers of the weak coupling constant. ${ }^{3}$ Notice that in this natural window, the fifth

\footnotetext{
${ }^{2}$ Unless the units explicitly appear, we work in the reduced Planck units where $M_{P} \equiv(8 \pi G)^{-1 / 2}=1$.

${ }^{3}$ Of course, for generic values of parameters the radiatively-denegated equivalence-violating couplings have to be properly tuned.
} 
force is close to its experimental limits and is potentially testable. Thus, in a framework in which neutrino superluminality is environmentally-induced, the fifth force is a natural consequence of the scenario.

The scale $M_{*}$ is further constrained by astrophysical and cosmological bounds coming from the star-cooling, and the Big Bang Nucleosynthesis (BBN), see [6]. The requirement that production of $h_{\mu \nu}$ in the stars gives a negligible correction to the cooling rate, implies an approximate bound, $M_{*}>10^{7-8} \mathrm{GeV}$. The cosmological bound is derived by requiring that the production rate of $h_{\mu \nu}$ during BBN, $\Gamma \sim T_{N}^{3} / M_{*}^{2}$, is subdominant to the expansion rate of the Universe, $H \sim T_{N}^{2} / M_{P}$. This implies the bound, $M_{*}>\sqrt{M_{P} T_{N}} \sim 10^{7-8} \mathrm{GeV}$, where we have taken BBN temperature to be $T_{N} \sim 10 \mathrm{MeV}$. Interestingly, the two bounds are very close, which is similar to situation [7] with analogous bounds on Kaluza-Klein graviton production in large extra dimensional scenario [8]. Note that these bounds $M_{*}>$ $10^{8} \mathrm{GeV}=10^{-10} M_{P}$ combined with (9) imply that $M<10^{6} M_{P}$. Thus the window for $M_{*}$ is $10^{-10} M_{P}<M_{*}<10^{-6} M_{P}$.

Thus, we have shown that additional asymmetric gravity-type force can provide environmental explanation of neutrino superluminality. We wish now to make a stronger statement and show that under the assumption of weak-coupling such a force is the only possibility. The argument goes in the following way. The environmental explanation by default implies that superluminality results from an effective background metric $g_{\mu \nu}^{(\nu)}$ caused by the environment. Since the effect is small, this effective metric can be represented as a small deviation from the flat metric

$$
g_{\mu \nu}^{(\nu)}=\eta_{\mu \nu}+\delta g_{\mu \nu}^{(\nu)}
$$

Since by assumption the theory is in a weak-coupling regime and perturbations are shortrange and local, the effective metric perturbation can be expanded in terms of canonically normalized massive degrees of freedom characterized by representations of the Poincaré group. The most general, up to two-derivative, linear expansion has the following form ${ }^{4}$

$$
\delta g_{\mu \nu}^{(\nu)}=\frac{h_{\mu \nu}}{M_{*}}+\eta_{\mu \nu} \frac{\phi}{M_{0}}+\frac{\partial_{\mu} \partial_{\nu} \phi}{M_{0}^{\prime 3}}+\frac{\partial_{\mu} A_{\nu}+\partial_{\nu} A_{\mu}}{M_{1}^{2}},
$$

where $h_{\mu \nu}, \phi$ and $A_{\mu}$ contain massive spin-2, spin-0 and spin-1 degrees of freedom respectively and $M_{0}, M_{0}^{\prime}, M_{1}$ are some mass scales. The degrees of freedom that appear with derivatives do not contribute to couplings with the conserved source at the linear level. This leaves us with spin- 2 and non-derivatively coupled spin-0 only. However, the coupling of spin-0 is proportional to $\eta_{\mu \nu}$, and thus, at the linear level no superluminality can be induced by coupling to $\phi$. This leaves us with the above-discussed spin-2 option.

An important open question is of course existence of a sensible UV-completion for such class of theories, cf. [10]. We have seen that accommodation of neutrino superluminallity imposes a non-universal sign coupling of a new spin-2 state. It is unclear whether such non-universally-coupled spin-2 states can be embedded in a consistent microscopic theory.

\footnotetext{
${ }^{4}$ Similarly to [9] we could include nonlinear terms like $\partial_{\mu} \phi \partial_{\nu} \phi$. However, these terms would lead to a higher order interaction and correspondingly to a lower strong-coupling scale.
} 
We have not addressed this issue in the present work, but we have to note, that should neutrinos be experimentally proven to be superluminal, the analogous question with be unavoidable for any effective theory that addresses such superluminality. ${ }^{5}$ Note that superluminality does not necessary lead to a breakdown of such a basic notion as causality, see e.g. [11] cf. [10]

In connection with UV-completion one can ask whether our $h_{\mu \nu}$ field can be identified with a Kaluza-Klein excitation(s) of large extra dimension to which neutrino can propagate. $^{6}$ Such a setup was already suggested as a possible origin of small neutrino masses [16-18]. This idea exploits the fact that the right handed neutrino is the only gauge-neutral particle and can naturally live in extra space, and thus share very weakcoupling properties with the graviton. In such a scenario neutrino naturally experiences different couplings with bulk gravity as compared to other standard model species. It is tempting to identify our spin-2 field $h_{\mu \nu}$ with one of (or the entire tower) of massive bulk Kaluza-Klein states. Of course, correspondingly the size of extra dimension has to be chosen to be comparable to earth's radius, perhaps along the lines of construction given in [6]. The scale $M_{*}$ then must originate from the wave-function overlap integral between neutrino and Kaluza-Klein species. Having this overlap the sign opposite to the ordinary graviton requires a very peculiar wave-function profiles, and currently we are not aware of any stable geometry of the extra dimensional space that could deliver it. This fact can be added as one particular difficulty for UV-completion. So the question of such an embedding will not be answered in the present work.

So far we have considered the coupling of the massive spin-2 field to the energymomentum tensor of a free and masseless neutrino field. Due to the Standard Model interactions this incomplete energy-momentum tensor is not conserved. This non-conservation introduces derivative couplings of the longitudinal component $h_{\mu \nu}^{\mathrm{LL}}$ of the massive spin2 field:

$$
h_{\mu \nu}^{\mathrm{LL}}=\varphi \eta_{\mu \nu}+\frac{\partial_{\mu} \partial_{\nu} \varphi}{m^{2}}
$$

to the $Z$ and $W^{ \pm}$bosons, neutrino $\nu$ and the corresponding lepton $\ell_{\nu}$, like e.g.:

$$
\mathrm{g} \frac{\partial_{\mu} \varphi \partial^{\mu} \bar{\nu} \gamma^{\alpha} \ell_{\nu} W_{\alpha}^{+}}{m^{2} M_{*}}
$$

and

$$
\frac{\mathrm{g}}{\cos \theta_{\mathrm{w}}} \frac{\partial_{\mu} \varphi \partial^{\mu} \bar{\nu} \gamma^{\alpha} \nu Z_{\alpha}}{m^{2} M_{*}}
$$

where $\mathrm{g}$ is the weak coupling constant and $\theta_{\mathrm{w}}$ is the Weinberg angle. These derivative interactions do not change the neutrino front velocity because the gauge bosons have vanishing vacuum expectation value. However, these irrelevant operators introduce a strongcoupling scale

$$
\Lambda=\left(m^{2} M_{*}\right)^{1 / 3},
$$

\footnotetext{
${ }^{5}$ One can turn the above argument around, and use the absence of UV-completion as the evidence against superluminal propagation. But then there would be no reason to write this note to start with.

${ }^{6}$ Apparent superluminality of neutrinos due to the propagation in an extra dimension was also considered in [12-14]. It can also happen that the speed of light in the bulk is larger than in our brane, see e.g. [15]. Again, in $4 \mathrm{~d}$ effective field theory language these options reduce to the scenario described above.
} 
which happens to coincide with the scale of Dark Energy for our choice of parameters:

$$
\Lambda=M_{P}\left(\frac{\ell_{P}}{r}\right)^{2 / 3}\left(\frac{M_{*}}{M_{P}}\right)^{1 / 3} \sim 10^{-3} \mathrm{eV}
$$

After the first version of this note appeared on arXiv e-Print server the neutrino superluminality was, in particular, confronted with: i) too strong neutrino energy loss due to bremsstrahlung of electron-positron pairs [19]; and ii) the pion decay kinematics [20]. Unfortunately, in all these processes, at the interesting energy range, the scalar graviton $\varphi$ quanta can not only be very efficiently emitted by neutrino but can also be absorbed from the condensate of $\varphi$ - background which is induced by the earth. The rather low strongcoupling scale (16) for these decay and absorption channels invalidates the perturbative calculations for the bremsstrahlung and requires a revision of the results on the pion decay kinematics. However, a detailed analysis of these effects goes beyond the purpose of this short note. Although our setup enders the effects of [19] and [20] un-calculable in weakcoupling, our analysis is complementary, since it opens up an universal gravitational side of the problem.

Here we would like to stress that, for any particle species, a non-fundamental modification of their front velocity (of the effective metric where they propagate) with respect to the speed of light (gravitational metric): a) can only occur in theories with irrelevant, nonrenormalizable operators and b) can only be caused by a spontaneous breaking of the Lorentz invariance. These irrelevant, nonrenormalizable operators introduce a strongcoupling scale. Thus any change of the front velocity is necessarily accompanied with a novel strong coupling scale which was not present in the standard model.

To conclude we have investigated an idea that superluminality of neutrinos can be a local environmental effect. We have shown that model-independently this would imply the existence of a new gravity-type field that is sourced by the earth and creates an effective superluminal metric for neutrinos. This follows fro the uniqueness of the mode-expansion of effective metric perturbation on any asymptotically Poincaré-invariant background. We have seen that treated as an effective low energy field theory such a setup passes all the immediate tests and may avoid other, more involved, and more recent phenomenological constraints. However, as we have demonstrated, the price to pay for the environmental neutrino superluminality is rather high. In particular, it necessarily includes: i) stronglycoupled physics on scales tremendously lower than a few $\mathrm{TeV}$ - the lowest cutoff scale for the currently known physics, ii) the sign-asymmetry in the coupling of massive graviton. We identify the latter fact as the main obstacle for a consistent UV-completion. While the too low strong-coupling scale results in the partial loss of calculability for a subsector of the standard model.

On phenomenological front this setup results into a natural prediction of a gravity-type force of an approximately planetary range. Without any proper adjustment, such a force is expected to be isotope-dependent, and thus, could be tested in precision gravitational experiments that look for equivalence-principle-violating forces. 


\section{Acknowledgments}

We thank Lasha Berezhiani, Fedor Bezrukov, Giga Chkareuli, Cesar Gomez and Goran Senjanovic for discussions. The work of G.D. was supported in part by Humboldt Foundation under Alexander von Humboldt Professorship, by European Commission under the ERC advanced grant 226371, and by the NSF grant PHY-0758032.

Open Access. This article is distributed under the terms of the Creative Commons Attribution License which permits any use, distribution and reproduction in any medium, provided the original author(s) and source are credited.

\section{References}

[1] OPERA collaboration, T. Adam et al., Measurement of the neutrino velocity with the OPERA detector in the CNGS beam, arXiv:1109.4897 [INSPIRE].

[2] G. Cacciapaglia, A. Deandrea and L. Panizzi, Superluminal neutrinos in long baseline experiments and SN198\%a, JHEP 11 (2011) 137 [arXiv:1109.4980] [INSPIRE].

[3] G. Amelino-Camelia et al., OPERA-reassessing data on the energy dependence of the speed of neutrinos, Int. J. Mod. Phys. D 20 (2011) 2623 [arXiv:1109.5172] [INSPIRE].

[4] A. Vainshtein, To the problem of nonvanishing gravitation mass, Phys. Lett. B 39 (1972) 393 [INSPIRE].

[5] C. Talmadge, J. Berthias, R. Hellings and E. Standish, Model independent constraints on possible modifications of newtonian gravity, Phys. Rev. Lett. 61 (1988) 1159 [INSPIRE].

[6] G. Dvali, G. Gabadadze, M. Kolanovic and F. Nitti, The power of brane induced gravity, Phys. Rev. D 64 (2001) 084004 [hep-ph/0102216] [INSPIRE].

[7] N. Arkani-Hamed, S. Dimopoulos and G. Dvali, Phenomenology, astrophysics and cosmology of theories with submillimeter dimensions and TeV scale quantum gravity,

Phys. Rev. D 59 (1999) 086004 [hep-ph/9807344] [INSPIRE].

[8] N. Arkani-Hamed, S. Dimopoulos and G. Dvali, The hierarchy problem and new dimensions at a millimeter, Phys. Lett. B 429 (1998) 263 [hep-ph/9803315] [INSPIRE].

[9] C.S. Gauthier, R. Saotome and R. Akhoury, Interaction of neutrinos with a cosmological K-essence scalar, JHEP 07 (2010) 062 [arXiv:0911.3168] [INSPIRE].

[10] A. Adams, N. Arkani-Hamed, S. Dubovsky, A. Nicolis and R. Rattazzi, Causality, analyticity and an IR obstruction to UV completion, JHEP 10 (2006) 014 [hep-th/0602178] [INSPIRE].

[11] E. Babichev, V. Mukhanov and A. Vikman, $k$-Essence, superluminal propagation, causality and emergent geometry, JHEP 02 (2008) 101 [arXiv:0708.0561] [INSPIRE].

[12] H. Pas, S. Pakvasa and T.J. Weiler, Sterile-active neutrino oscillations and shortcuts in the extra dimension, Phys. Rev. D 72 (2005) 095017 [hep-ph/0504096] [INSPIRE].

[13] J. Dent, H. Pas, S. Pakvasa and T.J. Weiler, Neutrino time travel, arXiv:0710.2524 [INSPIRE].

[14] S. Hollenberg, O. Micu, H. Pas and T.J. Weiler, Baseline-dependent neutrino oscillations with extra-dimensional shortcuts, Phys. Rev. D 80 (2009) 093005 [arXiv: 0906. 0150] [InSPIRE]. 
[15] E. Kiritsis, Supergravity, D-brane probes and thermal super Yang-Mills: a comparison, JHEP 10 (1999) 010 [hep-th/9906206] [INSPIRE].

[16] N. Arkani-Hamed, S. Dimopoulos, G. Dvali and J. March-Russell, Neutrino masses from large extra dimensions, Phys. Rev. D 65 (2002) 024032 [hep-ph/9811448] [INSPIRE].

[17] K.R. Dienes, E. Dudas and T. Gherghetta, Neutrino oscillations without neutrino masses or heavy mass scales: a higher dimensional seesaw mechanism, Nucl. Phys. B 557 (1999) 25 [hep-ph/9811428] [INSPIRE].

[18] G. Dvali and A.Y. Smirnov, Probing large extra dimensions with neutrinos, Nucl. Phys. B 563 (1999) 63 [hep-ph/9904211] [INSPIRE].

[19] A.G. Cohen and S.L. Glashow, Pair creation constrains superluminal neutrino propagation, Phys. Rev. Lett. 107 (2011) 181803 [arXiv:1109.6562] [INSPIRE].

[20] R. Cowsik, S. Nussinov and U. Sarkar, Superluminal neutrinos at OPERA confront pion decay kinematics, Phys. Rev. Lett. 107 (2011) 251801 [arXiv:1110.0241] [INSPIRE]. 